\title{
Primary gastrointestinal stromal tumour of the ileum pre-operatively diagnosed as an abdominal abscess
}

\author{
PATRIZIA RUBINI and FRANCESCO TARTAMELLA \\ Department of Surgery, Institute of General Surgery, University of Parma, I-43100 Parma, Italy
}

Received May 12, 2016; Accepted July 15, 2016

DOI: $10.3892 /$ mco.2016.1009

\begin{abstract}
The present case report described the acute presentation, diagnosis and management of a primary gastrointestinal stromal tumour (GIST) of the ileum. A male patient (age, 51 years) was admitted to Maggiore Hospital (Parma, Italy) due to presenting with fever, dysuria and lower abdominal pain. Ultrasonography and computed tomography showed a 7,5x5,5-cm pelvic mass containing air and purulent fluid indicative of an intraperitoneal abscess. The patient was subjected to diagnostic laparoscopy, which revealed a huge, soft cystic mass arising from the small bowel. The procedure was then converted to an open exploration through a midline incision. Ileal resection including a Meckel's diverticulum was performed. Macroscopic examination revealed that the cystic mass was filled with a large amount of pus, probably due to communication between the tumour mass and the small bowel lumen. In fact, the surgical specimen showed enteric leakage from the ileal mucosal ulcer into the tumour mass. Histopathology and immunohistochemistry of the abscess wall identified a spindle-cell mesenchymal-type, c-KIT-positive neoplasm. The post-operative course was uneventful and adjuvant imatinib mesylate was administered for 1 year. Follow-up by computed tomography demonstrated no tumour recurrence at 72 months after surgery.
\end{abstract}

\section{Introduction}

Gastrointestinal stromal tumours (GISTs) are mesenchymal cell-derived neoplasms of the gut that have assumed oncological importance out of proportion to their relatively rare incidence due to having provided important insight into cancer biology. They originate from the interstitial cells of Cajal,

Correspondence to: Professor Patrizia Rubini, Department of Surgery, Institute of General Surgery, University of Parma, via Gramsci 14, I-43100 Parma, Italy

E-mail: patrizia.rubini@unipr.it

\section{Abbreviation: GIST, gastrointestinal stromal tumour}

Key words: abdominal abscess, gastrointestinal stromal tumour, acute abdomen the pacemaker cells that regulate peristalsis in the digestive tract (1). The pathogenesis of GISTs is based on the oncogenic, mutational activation of KIT tyrosine kinase in $>95 \%$ of cases, or less frequently, on that of platelet-derived growth factor receptor alpha $(2,3)$.

Pre-clinical studies revealed that the drug imatinib mesylate inhibits the activity of the protein products of these two GIST-associated oncogenes, which led to the development of one of the first identified pathway-driven, highly effective treatments for a solid tumour. While the majority of GISTs are chemotherapy-resistant, $>50 \%$ of affected patients responded to imatinib mesylate, increasing the average survival of patients with advanced disease to 5 years (4).

GISTs are rare with an age-adjusted yearly incidence rate of 0.68/100,000 individuals; they mostly affect individuals aged $>50$ years with a male-to-female ratio of 1.5 (5). GISTs occur throughout the gastrointestinal tract from the lower esophagus to the anus with the most common sites being the stomach (60\%), jejunum and ileum (30\%), duodenum $(5 \%)$ and colorectum $(<5 \%)(6)$.

Small bowel GISTs have a wide clinicopathological spectrum at presentation, ranging from minute incidental nodules to large tumours. Clinical signs and symptoms are usually non-specific, and acute abdomen prompting emergency surgery is rare and commonly arises from bleeding, intestinal obstruction, tumour rupture with intra-abdominal haemorrhage, pelvic mass and appendicitis-like acute pain $(6,7)$.

The present study reported on a case of a primary small bowel GIST, which was initially diagnosed as an abdominal abscess. While this complication has been reported in the literature (6-9), abscess formation inside a GIST tumour mass is a rare occurrence.

\section{Case report}

A male patient (age, 51 years) was admitted to Maggiore Hospital (Parma, Italy), who had been presenting with fever, dysuria and lower abdominal pain for four days. On admission, the patient's axillary temperature was $39,4^{\circ} \mathrm{C}$, the heart rate was 104 beats per minute and the blood pressure was 145/90 mmHg. The patient did not have neurofibromatosis type I or any other relevant family history, was taking no medications and his past medical history was unremarkable. On physical examination, the upper abdomen was soft with normal sensitivity, while in the lower abdomen, a moderate 
tenderness in the hypogastrium without peritoneal signs was present. General physical examination was unremarkable.

Routine laboratory data on admission revealed a white blood cell count of $18,200 / \mathrm{mm}^{3}$ (normal range, $3500-11,000 / \mathrm{mm}^{3}$ ), C-reactive protein levels of $130 \mathrm{mg} / \mathrm{l}$ (normal range, $0-10 \mathrm{mg} / \mathrm{l}$ ) and an erythrocyte sedimentation rate of $65 \mathrm{~mm} / \mathrm{h}$ (normal range, $0-22 \mathrm{~mm} / \mathrm{h}$ ). Other biochemical parameters and liver function tests were normal. Plain abdominal radiography showed an extraintestinal air-fluid level in the hypogastric region without any free intraperitoneal air. Ultrasonography and computed tomography (CT) of the abdomen revealed the presence of a 7,5x5,5 cm pelvic mass containing air and liquid, which was located behind the bladder and close to a normal appendix, and was indicated to be an intraperitoneal abscess. Gastrografin enema examination excluded colon pathologies.

Diagnostic laparoscopy showed a huge, soft cystic mass adherent to the small bowel. The procedure was then converted to an open exploration through a midline incision. At laparotomy, the cyst-like mass arising from the ileal wall, with a prevalent extramural growth and mostly protruding into the mesentery, was located $\sim 40 \mathrm{~cm}$ from the ileo-cecal valve. A normal Meckel's diverticulum was observed at $\sim 20 \mathrm{~cm}$ from the mass. No other visceral abnormalities or intraperitoneal fluids were detected. Ileal resection, including the Meckel's diverticulum, and end-to-end ileal anastomosis were performed without tumour rupture.

At macroscopic examination, the excised mass, measuring $7 \mathrm{~cm}$ in diameter (Fig. 1), showed a cystic aspect with a central cavity containing a large amount of pus (Fig. 2); closer examination revealed a communication with the small bowel lumen via a mucosal ulceration and enteric leakage through the central part of the mass (Fig. 3).

Histopathology showed a spindle-cell mesenchymal-type neoplasm with erosion of the small bowel mucosal layer. Immunohistochemistry revealed that tumour cells were c-KIT-positive, CD34-negative, $\alpha$-smooth muscle actin-negative and S-100-negative. The mitotic rate was $<5 / 50$ high-power fields. The resection margins and mesenteric lymph nodes were disease free. Pathological examination of the Meckel's diverticulum was unremarkable. Of note, histopathology of the abscess wall showed that tumour cells had replaced the full thickness of the ileal wall. The final diagnosis was intermediate-risk GIST.

The post-operative course was uneventful and the patient was discharged home on the seventh post-operative day. Adjuvant imatinib mesylate (400 mg daily for 1 year) was administered. Yearly follow-up by CT scan was performed and at 72 months after surgery, no tumour recurrence had been detected.

\section{Discussion}

Among the acute clinical presentations of primary small bowel GIST, tumour mass perforation with peritonitis has been infrequently reported in the literature, while abscess formation inside the GIST mass represents an exceptional occurrence (6-9). In fact, a literature search of the PubMed database on sporadic primary small bowel GISTs including studies published from January 1976 to December 2015 in

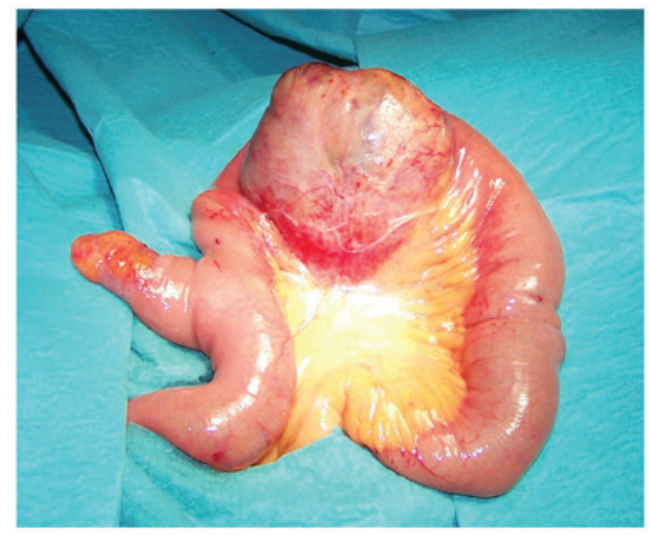

Figure 1. Gross appearance of the cystic gastrointestinal stromal tumour.

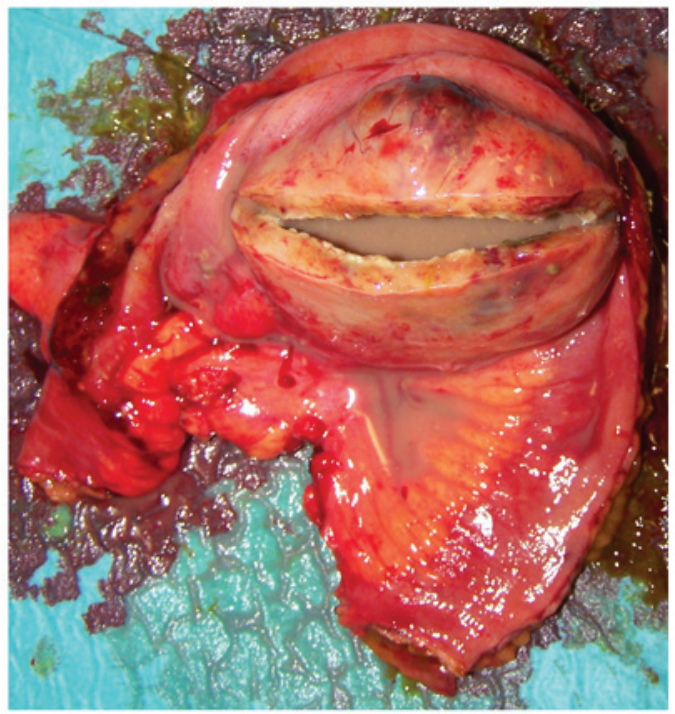

Figure 2. Incision in the gastrointestinal stromal tumour wall revealing a large quantity of pus.

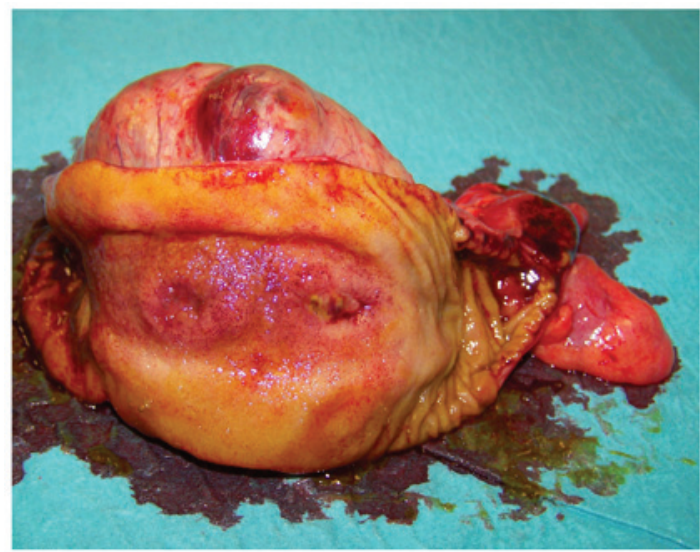

Figure 3. Segment of the ileal wall showing a fistula orifice between the tumour and gut lumen arising from bowel mucosal ulceration.

English language using the search terms 'gastrointestinal stromal tumour' (7,595 studies) and 'gastrointestinal stromal tumour complications' (1,545 studies) retrieved only two cases of abscess formation inside a small bowel GIST $(10,11)$. 
The first case study by Bardell et al (10) reported on a large GIST of the ileum in a male patient (age, 41 years), presenting as an infected mesenteric cystic mass. The patient was subjected to ileal resection including the mass. Pathological examination of the resected specimen showed a large GIST with a central cystic area communicating with the intestinal lumen via a fistula tract. The second case was a male patient (age, 22 years) with abdominal pain and fever as well as a pelvic mass detected by CT, who was subjected to CT-guided drainage of the abscess and subsequently to segmental resection of the jejunum with subsequent pathological examination revealing a GIST (11). In these two cases as well as in the case of the present study, it is likely that a wide coagulative necrosis inside the tumour mass caused ulceration of the intestinal mucosa with enteric leakage and abscess development; in fact in the present study, histopathological analysis of the abscess wall showed that tumour growth had replaced the full thickness of the gut wall. By contrast, in the few reported cases of GIST complicated by an abscess, the abscess wall was constituted by omentum and surrounding intestinal loops wrapping a GIST tumour mass perforation $(8,12)$.

The macroscopic presentation of GIST in the present study represents an exceptional finding and differential diagnoses included abdominal cystic masses such as infected mesenteric cysts of neoplastic or lymphatic origin, enteric duplication cysts and cystic teratomas $(13,14)$. However, histopathology revealed positive staining for $\mathrm{c}-\mathrm{KIT}$, which excluded the diagnosis of other types of mesenchymal neoplasm.

The prognosis for patients with GISTs is difficult to predict. The most powerful and most widely examined criteria for evaluating the biological properties of GIST are tumour size and mitotic activity (15). The impact of the anatomical location is controversial, while the majority of studies indicate that primary small bowel GISTs have a more aggressive behaviour than gastric GISTs with similar size and mitosis parameters $(6,7,16)$. Moreover, tumour rupture, either occurring spontaneously or at surgery, is considered an additional negative prognostic factor due to increasing the risk of intra-abdominal implant tumours (17).

In conclusion, the present study reported on a patient who appeared to have an abdominal abscess, which, after surgical resection, was revealed to be a GIST including an abscess. While this pathology is rare, resection of a preliminarily diagnosed abdominal abscesses should be performed with caution to avoid the rupture of a possible tumour and peritoneal seeding. In the present case, conversion from laparotomy to open surgery was mainly performed due to tumour mass size and tumour wall weakness. The laparoscopic approach should be considered, taking into account the result of the intraoperative assessment, the diameter of the mass, the type of tumour wall and the feasibility of successful oncologic clearance.

\section{References}

1. Kindblom LG, Remotti HE, Aldenborg F and Meis-Kindblom JM: Gastrointestinal pacemaker cell tumor (GIPACT): Gastrointestinal stromal tumors show phenotypic characteristics of the interstitial cells of Cajal. Am J Pathol 152: 1259-1269, 1998.

2. Hirota S, Isozaki K, Moriyama Y, Hashimoto K, Nishida T, Ishiguro S, Kawano K, Hanada M, Kurata A, Takeda M, et al: Gain-of-function mutations of c-kit in human gastrointestinal stromal tumors. Science 279: 577-580, 1998.

3. Heinrich MC, Corless CL, Duensing A, McGreevey L, Chen CJ, Joseph N, Singer S, Griffith DJ, Haley A, Town A, et al: PDGFRA activating mutations in gastrointestinal stromal tumors. Science 299: 708-710, 2003.

4. Demetri GD, von Mehren M, Blanke CD, Van den Abbeele AD, Eisenberg B, Roberts PJ, Heinrich MC, Tuveson DA, Singer S, Janicek M, et al: Efficacy and safety of imatinib mesylate in advanced gastrointestinal stromal tumors. N Engl J Med 347: 472-480, 2002.

5. Tran T, Devila JA and El-Serag HB: The epidemiology of malignant gastrointestinal stromal tumors: An analysis of 1,458 cases from 1992 to 2000 . Am J Gastroenterol 100: 162-168, 2005.

6. Miettinen M and Lasota J: Gastrointestinal stromal tumors: Review on morphology, molecular pathology, prognosis, and differential diagnosis. Arch Pathol Lab Med 130: 1466-1478, 2006.

7. Miettinen M, Makhlouf $\mathrm{H}$, Sobin LH and Lasota J: Gastrointestinal stromal tumors of the jejunum and ileum: A clinicopathologic, immunohistochemical, and molecular genetic study of 906 cases before imatinib with long-term follow-up. Am J Surg Pathol 30: 477-489, 2006.

8. Lord C, Ozgediz D and Cohen MJ: Image of the month. Gastrointestinal stromal tumor. Arch Surg 144: 87-88, 2009.

9. Efremidou EI, Liratzopoulos N, Papageorgiou MS and Romanidis K: Perforated GIST of the small intestine as a rare cause of acute abdomen: Surgical treatment and adjuvant therapy. Case report. J Gastrointestin Liver Dis 15: 297-299, 2006.

10. Bardell T, Jalink DW, Hurlbut DJ and Mercer CD: Gastrointestinal stromal tumour: Varied presentation of a rare disease. Can J Surg 49: 286-289, 2006.

11. Chen HW and Lin TY: Tumor abscess formation caused by Morganella morganii complicated with bacteremia in a patient with gastrointestinal stromal tumor. Clin Res Hepatol Gastroenterol 36: e29-e31, 2012.

12. Karagülle E, Türk E, Yildirim E, Gõktürk HS, KiYici H and Moray G: Multifocal intestinal stromal tumors with jejunal perforation and intra-abdominal abscess: Report of a case. Turk J Gastroenterol 19: 264-267, 2008.

13. Dow N, Giblen G, Sobin LH and Miettinen M: Gastrointestinal stromal tumors: Differential diagnosis. Semin Diagn Pathol 23: 111-119, 2006.

14. Rubin BP: Gastrointestinal stromal tumours: An update. Histopathology 48: 83-96, 2006.

15. Fletcher CD, Berman JJ, Corless C, Gorstein F, Lasota J, Longley BJ, Miettinen M, O'Leary TJ, Remotti H, Rubin BP, et al: Diagnosis of gastrointestinal stromal tumors: A consensus approach. Hum Pathol 33: 459-465, 2002.

16. Miettinen $\mathrm{M}$ and Lasota J: Gastrointestinal stromal tumors: Pathology and prognosis at different sites. Semin Diagn Pathol 23: 70-83, 2006.

17. Takahashi T, Nakajima K, Nishitani A, Souma Y, Hirota S, Sawa Y and Nishida T: An enhanced risk-group stratification system for more practical prognostication of clinically malignant gastrointestinal stromal tumors. Int J Clin Oncol 12: 369-374, 2007. 